\title{
Computational fluid dynamics design and forecasting of urban drainage unit operation behaviour
}

\author{
J. Sansalone \\ University of Florida, USA
}

\begin{abstract}
A primary and historical consideration of urban drainage unit operation behavior is the transport/fate (control) of particulate matter (PM). This consideration has a strong foundation given that PM is the primary source and sink for chemicals (metal elements, organics, nutrients...) and a primary habitat as well as vehicle for pathogens. However the control of PM is challenging; in part due to the heterodisperse and temporally variable size gradation, complex geometries of many unit operations and highly variable and episodic flow rates. The control of PM has led to a spectrum of unit operations from hydrodynamic separation (HS) for coarse particulate and gross detritus separation that do not provide hydrologic control, to large clarification systems such as wet or dry storage systems that provide a much wider clarification response and potential hydrologic control. This study applies the principles of computational fluid dynamics (CFD) to predict PM clarification behavior of these systems subject to dilute multiphase flows $(<2 \% \mathrm{PM})$, typical of urban drainage. A standard turbulence model is used to resolve flow, and a discrete phase model (DPM) is utilized to examine PM clarification response. CFD results closely followed physical model results across the entire range of flow rates. Results demonstrate that for smaller unit operations such as an HS that scour can result in significant degradation of behavior that are not maintained. Comparisons are made between measured and CFD modeled results and mass balances are identified. CFD have become one of the most powerful tool for design and forecasting of urban drainage unit operation behavior since continuous simulation modelling.
\end{abstract}

Keywords: computational fluid dynamics (CFD), urban drainage, best management practices (BMPS), clarification, particulate matter, sedimentation. 


\section{Introduction}

The fate and transport of PM and PM-associated chemicals/pathogens in urban drainage is complex due to the coupling of unsteady hydrology and size heterodisperse PM (Sansalone [1]). Studies have reported on small-footprint unit operations (UOs), sometimes termed best management practices (BMP) when applied to stormwater, for control of PM and PM-associated chemicals. Physical modeling of hydrodynamic separators (HS) by Kim and Sansalone [2] as well as storage basins and filters have been conducted (Sansalone 1999 [3]).

Many of these UOs are tested based on steady flows and particle size distributions (PSD). Such steady loadings, appropriate for wastewater treatment are an initial basis to examine mechanistic behavior, but must be coupled with unsteady testing and evaluation of scour and maintenance frequency given that the performance of many current BMP systems can deteriorate in a period as short as a month without maintenance. Rainfall-runoff models such as the Stormwater Management Model (SWMM) (Huber and Dickinson [4]) allow complex coupling of hydrology, PM and PM-bound transport, but do not yet account for BMPspecific calibration, maintenance, scaling or misbehavior. Among approaches to predict HS performance, Weib [5] applied overflow theory and Paul et al. [6] utilized semi-empirical equations of vortex flow behavior. Similarly, for filtration, granular filters and permeable pavement filter performance have been examined (Keblin et al. [7]). While these approaches have specific advantages, development of multiphase computational fluid dynamics (CFD) (Dickenson and Sansalone [8]) offers a general and dynamic tool to predict and analyze coupled hydrodynamics and PM dynamics in BMPs such as the HS units and filters. Studies have applied CFD to treatment of combined sewer overflow (CSO) for storage chambers. CFD approaches have utilized a Reynolds Stress Model (RSM), (Faram and Harwood [9]) for turbulent flows and a Lagrangian Discrete Phase Model (DPM) for PM separation by a sediment interceptor. Tyack and Fenner [10] compared measured velocity profiles to simulation by a Renormalization Group (RNG) k- $\varepsilon$ turbulence model.

While such BMPs have been used for decades, addressing scour and maintenance practices with parallels to efforts to prevent channel scour has only been included recently as part of a comprehensive design and management approach (Gulliver et al. [11]). PM scour in BMPs is a function of many parameters including flow velocity, shear stress and stream power.

\section{Objectives}

With respect to the transport and fate of PM objectives are to illustrate a CFD approach coupled with physical modelling validation for common BMPs: a screened HS, a radial cartridge filter (RCF) and a storage basin functioning as a clarifier. BMPs are examined for steady-flow physical modelling and then with unsteady event-based transient hydrodynamics, PM transport. Scour is illustrated as well as the challenge of maintenance highlighted from physical modeling (Sansalone et al. [12]). 


\section{Materials and methods}

\subsection{Physical model testing for PM separation performance of HS}

In keeping with the physical mechanisms of the HS these units are described fundamentally as unit operations and the term "BMP" will be avoided. There are many incarnations of HS units, common classes include screened, baffled or combinations. All units are Type I gravitational settling units. This study illustrates a screened HS unit. In this testing all transport and fate analysis was conducted under clean bed conditions, except when scour was examined. Separation mechanism descriptions are available elsewhere (Pathapati and Sansalone [13]). The experimental setup consisted of a reservoir of influent potable water. The flow delivery system consisted of a centrifugal pump to achieve flow rates ranging from 0.16 to $23.40 \mathrm{~L} / \mathrm{s}$ which were monitored utilizing a $70 \mathrm{KHz}$ ultrasonic sensor to measure depth of flow in a $15.25 \mathrm{~cm}$ Parshall flume. An influent hetero-disperse PM gradation with a measured gravimetric median particle diameter $\left(\mathrm{d}_{50 \mathrm{~m}}\right)$ of 70 $\mu \mathrm{m}$ was utilized to prepare slurry that was then injected to the screened HS, on a constant gravimetric and granulometric basis, in order to maintain a constant influent PM of $200 \mathrm{mg} / \mathrm{L}$ as total suspended solids (TSS) measured as suspended sediment concentration (SSC). Separated PM was recovered as wet slurry, dried at $40{ }^{\circ} \mathrm{C}$, weighed and a mass balance check required a $90 \%$ mass balance with the PSD determined based on ASTM D422 (ASTM [14]).

\subsection{Physical model testing for scour of previously separated PM from HS}

Prior to each scour test the HS was cleaned and then pluviated with PM of known PSD to pre-determined depths corresponding to 50 and $100 \%$ of the capacity of the HS sump. The mixture used with the screened HS was poorly-graded sediment with a median particle diameter $\left(\mathrm{d}_{50 \mathrm{~m}}\right)$ of $110 \mu \mathrm{m}$. The HS was then filled with potable water at a low flow rate to minimize any re-suspension and to maintain clear supernatant $(<5 \mathrm{NTU})$ prior to the actual test. Flow rates were achieved through a pump and recirculation system. The total influent volume was maintained constant for each run and 20 duplicated effluent samples taken per run. SSC was measured for each sample. The magnitude of scour was subsequently evaluated by effluent mass load and effluent concentration. PSDs were measured with a laser diffraction analyzer.

\subsection{Physical model for PM separation by filtration}

There are many filter configurations; in this study a radial flow system was tested. Engineered oxide modified media with a specific gravity of 2.35 was utilized as the granular media. The median diameter of the media was measured by a digital imaging method was $3.56 \mathrm{~mm} \pm 0.8 \mathrm{~mm}$. The mean total clean bed porosity (porosity of the entire media bed) with the media was measured to be 0.71 . Internal media porosity was measured by porosimetry and found to be $0.37 \pm 0.022$. The physical model and analysis for controlled testing of the RCF is similar to the HS described in the previous section, excepting the influent PSD for the RCF was a 
non-cohesive silt with a mass-based $\mathrm{d}_{50 \mathrm{~m}}$ of $17 \mu \mathrm{m}$. Influent and effluent PSDs were measured by laser diffraction.

\subsection{Unsteady event-based physical model testing of a HS}

The area for unsteady event-based testing are two urban highway catchments in Baton Rouge, LA (USA) on I-10, with total contributing drainage area of $1088 \mathrm{~m}^{2}$. The Average Daily Traffic (ADT) was 142,000 total vehicles. Surface runoff was collected a sheet flow draining to a catch basin. A detailed description of the geography, instrumentation and sampling methods for real-time urban rainfallrunoff treatment by the screened HS is available elsewhere (Sheng et al. [15]). Full cross-sectional flow weighted sampling was performed at the inlet and the effluent outlet of the screened HS. Each rainfall-runoff event was routed through a precleaned flow conveyance system and screened HS.

\subsection{Design and forecasting of large-scale clarifier as a storage basin}

While physical models are ultimately needed for numerical model validation of PM transport/fate, tools such as CFD are valuable and economical for UO design and forecasting of behaviour under the intended range of event-based or continuous loadings. The behaviour of an internal retrofit for a $40,000 \mathrm{~m}^{3}$ storage basin as an on-line clarifier located in south Florida (USA) is illustrated. The basin is loaded by four separate inflow conveyances each from separate urban commercial, highway or airport land uses and the outflow is a triple box culvert. The retrofit design storm event was the mean annual event $(2.33$ year return storm). The internal retrofit was comprised of a series of permeable gabion baffles topped with a movable geosynthetic curtain that could be raised to the water surface as required. The retrofit basin forecasting with an unsteady CFD model was focused on PM, total phosphorus (TP) and total nitrogen (TN).

\subsection{Computational fluid dynamics (CFD) models}

A three dimensional (3D) approach was required for the HS and the RCF. The governing conservation equations for the continuous phase are a variant of the Navier-Stokes (N-S) equations, namely the Reynolds Averaged N-S (RANS) equations for a turbulent flow regime. These mass and momentum conservation equations for incompressible flows are briefly summarized herein. The RANS conservation equations are obtained from the $\mathrm{N}-\mathrm{S}$ equations by applying the Reynolds' decomposition of fluid flow properties into their time-mean value and fluctuating component. The mean velocity is defined as a time average for a period $t$ which is larger than the time scale of the fluctuations. The RANS equations for continuity and momentum conservation are summarized.

$$
\begin{gathered}
\frac{\partial}{\partial x_{i}}\left(\rho \overline{u_{i}}\right)=0 \\
\rho \frac{\partial \overline{u_{i}}}{\partial t}+\rho \frac{\partial}{\partial x_{j}}\left(\overline{u_{i} u_{j}}\right)+\rho \frac{\partial}{\partial x_{j}}\left(\overline{u_{j} u_{i}}\right)=-\frac{\partial \bar{p}}{\partial x_{i}}+\mu \frac{\partial^{2} \overline{u_{i}}}{\partial x_{j}{ }^{2}}+\rho g_{i}
\end{gathered}
$$


In the equations $\rho$ is fluid density, $x_{i}$ is $i^{\text {th }}$ direction vector, $u_{j}$ is Reynolds averaged velocity in $i^{\text {th }}$ direction; $p_{j}$ is the Reynolds averaged pressure; and $g_{i}$ is the sum of body forces in $i^{\text {th }}$ direction. Reynolds decomposition of the momentum equation generates a term originating from the nonlinear convection component from original equation; these Reynolds stresses are represented.

$$
-\rho \overline{u_{i}^{\prime} u_{j}^{\prime}} \text {. }
$$

Reynolds stresses contain information about the flow turbulence structure. Since Reynolds stresses are unknown, closure approximations can be made to obtain approximate solution of the equations. In this study the realizable k- $\varepsilon$ model is used to resolve the closure problem (Versteeg and Malalasekera [16]). While there are a range of turbulence models the standard k- $\varepsilon$ model has been illustrated as a robust approach and has been applied to hydrocyclones with swirling flows. The standard k- $\varepsilon$ model equations for k (turbulent kinetic energy) and $\varepsilon$ (rate of dissipation of k) area available elsewhere and not reproduced herein (Launder and Spalding [17]). The HS screen was modeled as a porous perforated plate with the addition of a momentum source term to the standard fluid flow equations.

The filter bed was modeled with a macroscopic approach by utilizing appropriate universal parameters such as the effective porosity $(\eta)$, inertial and viscous resistance coefficients. All parameters were calculated based on the assumption of homogeneity of media in terms of pore size distribution and media particle diameter. This was a reasonable assumption as the media was pluviated into the cartridge, and the median media was $3.56 \mathrm{~mm} \pm 0.8 \mathrm{~mm}$. The Ergun equation for filter bed flow; applicable across all flow regimes, was utilized.

$$
\boldsymbol{i}=\frac{\Delta \boldsymbol{H}}{\boldsymbol{L}}=\frac{\boldsymbol{k}_{0}\left(\boldsymbol{L}_{\boldsymbol{e}} / \boldsymbol{L}\right)^{2} \boldsymbol{\mu}}{\rho g} \frac{(1-\eta)^{2}}{\eta^{3}}\left(\frac{\boldsymbol{a}}{\boldsymbol{v}}\right)^{2} \boldsymbol{V}+\boldsymbol{k}_{2} \frac{1-\eta}{\eta^{3}}\left(\frac{a}{v}\right) \frac{V^{2}}{g}
$$

In this expression, $\Delta H / L=$ head loss per unit depth of media bed which is the hydraulic gradient, $i ; k_{0}=$ shape factor; $\mu=$ fluid viscosity $\left(\mathrm{N}-\mathrm{s} / \mathrm{m}_{2}\right) ; \rho=$ fluid density $(\mathrm{g} / \mathrm{cm} 3) ; g=$ acceleration of gravity $(9.81 \mathrm{~m} / \mathrm{s} 2) ; \eta=$ bed macro porosity (the pore volume between the packed media); $a / v=$ media surface area per unit of media volume $(\mathrm{m}-1) ; V=$ superficial velocity $(\mathrm{m} / \mathrm{s}) ; k_{2}=$ dimensionless Ergun constant $\left(k_{2}\right)(0.5$ for angular porous media). A Lagrangian particle tracking approach for dilute multiphase flows (particle volume fraction $\mathrm{F}<<0.1 \%$ ) was applicable. In this study, particles were tracked in a Lagrangian frame of reference, based on particle point of injection, particle morphology and grid density after modelling the flow field utilizing a Eulerian approach. The discrete phase model is based on a force balance based on Newtonian and Stokesian settling regimes. Analysis assumed spherical particles with settling parameters as a function of the Reynolds number. The tracking length of particles was determined based on hydraulic residence times calculated for neutrally buoyant tracer 'particles'. DPM boundary conditions included a 'reflect' boundary condition at all physical wall boundaries, and 'escape' boundary condition at the effluent outlet.

For scour, a steady flow field was established, by solving for flow with a k- $\varepsilon$ model. Particles were "injected" at a given point or uniformly across a surface. The PM velocity is zero at the surface of injection, and therefore, the particle 
motion from the point of injection is defined by the ambient fluid dynamics. A number of plane surfaces were created at regular intervals inside the sump. Particles were injected uniformly across these surfaces. Particle trajectories are obtained by integration of equations of motion for PM. Particles were defined at diameters associated with the measured sizes and a measured specific gravity. Particles eluted (washout) from the HS after integrating over the specified length were considered to have been scoured by the HS. The length of tracking particles was chosen based on the following criteria. At each plane surface, neutrally buoyant 'tracer' particles were first injected. These 'tracer' particles were iteratively tracked from the points of injection, until the maximum possible number of particles was eluted from the HS. The effluent load represented integration across the injection surfaces between the number of influent particles injected and the separated particles that are not eluted.

The computational geometry was discretized using an unstructured mesh with tetrahedral elements. Grid independence was achieved for the HS and RCF. This study utilized the finite volume method (FVM) for CFD. A cell-centered scheme was used in discretization and a second-order upwind scheme was utilized for computing values at cell faces. A cell-centered scheme was used in the process of discretization. The SIMPLE (Semi-Implicit Method for Pressure Linked Equations) algorithm accounted for pressure-velocity coupling. These criteria apply to transient modelling of the screened HS, with the exception of a transient SIMPLE algorithm for pressure-velocity coupling. The criterion for iterative convergence was set at $1 \times 10^{-3}$ for all simulations.

\section{Results and discussion}

\subsection{Physical and CFD model testing of HS and RCF}

Figure 1 illustrates effluent PM concentrations and PSDs from the HS and RCF, for an influent PM of $200 \mathrm{mg} / \mathrm{L}$ across the range of flow rates. Range bars represent standard deviations between replicated effluent samples. Effluent concentrations ranged from 64.5 to $117.6 \mathrm{mg} / \mathrm{L}$ across flow rates ranging from 1.59 to $19.87 \mathrm{~L} / \mathrm{s}$. The PSD of PM eluted from the HS is illustrated in Figure 1. The separation ability of the HS is limited to only very coarse PM. Figure 1 also illustrate CFD forecasting of effluent PM and PSD. Model forecasting agrees with measured data and the absolute relative percent difference (RPD) was used as an index of comparison. Figure 2 illustrates the physical models for the HS and RCF.

\subsection{Physical and CFD model of HS for scour of deposited PM}

The potential for re-suspension and scour of preloaded PM in the HS was examined as a function of flow and as a function of PM depth in the HS sump and volute chambers. Measured and CFD modeled effluent PSDs are compared in Figure 3. The CFD model forecasts effluent PM gradations with a clear dependence of scour on the PSD of preloaded PM. The smallest PM not scoured is approximately $120 \mu \mathrm{m}$, noting the $\mathrm{d}_{50 \mathrm{~m}}$ of the poorly-graded PSD is $100 \mu \mathrm{m}$. 

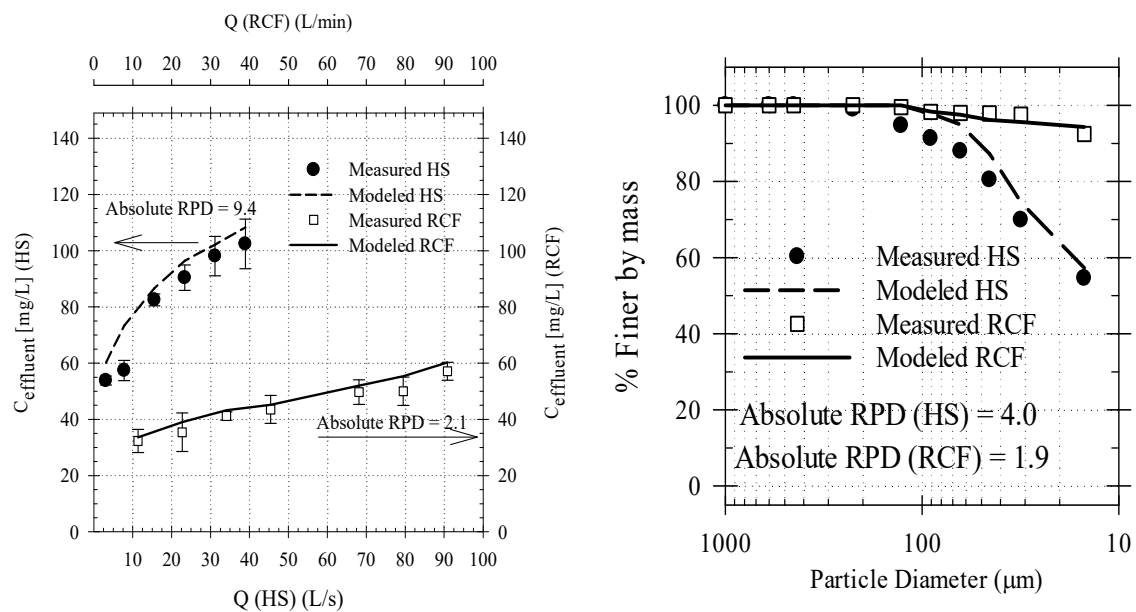

Figure 1: Measured and CFD modeled effluent PM and PSD for HS and RCF.
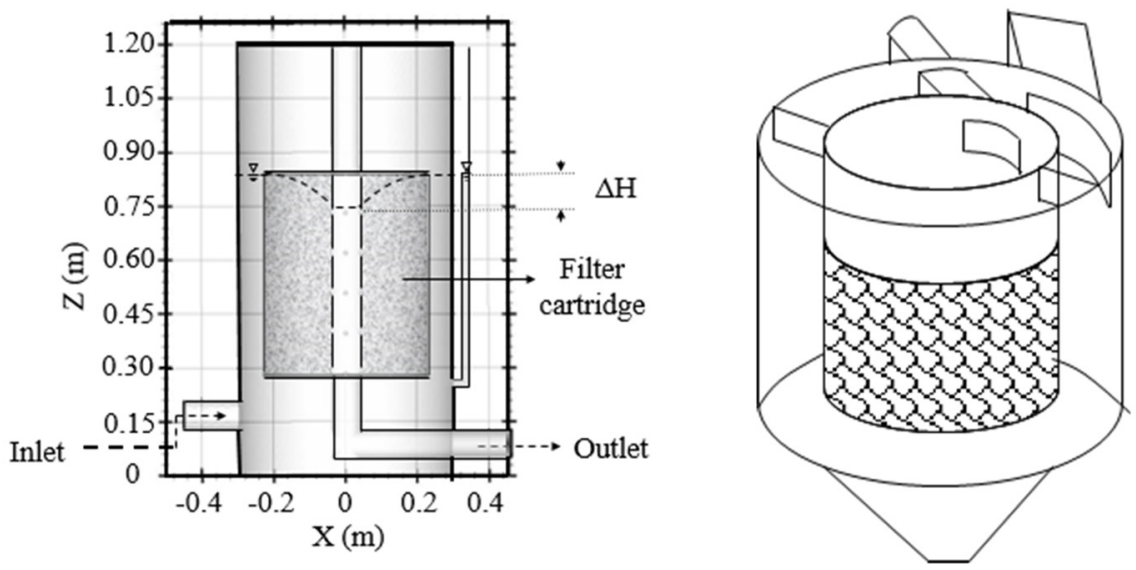

Figure 2: $\quad$ Physical model of RCF (left) and $1 \mathrm{~m}$ diameter x $1 \mathrm{~m}$ high HS (right).

\subsection{Uncontrolled physical and CFD model testing of HS on an event basis}

The 20 August 2004 rainfall-runoff event was a high intensity, short duration storm. Figure 4 illustrates the hydrograph and hyetograph of the rainfall-runoff event. The average flow rate was $5.1 \mathrm{~L} / \mathrm{s}$ and a total volume of $12.29 \mathrm{~m}^{3}$ was delivered. The influent PSD illustrated in Figure 4 was predominantly very coarse $\left(\mathrm{d}_{50}=300 \mu \mathrm{m}\right)$ and the total influent mass load was $10.59 \mathrm{~kg}$. The overall PM removal was $63 \%$ on a dry mass basis. The transient CFD model is in good agreement with measured effluent PSDs with an absolute RPD less than $10 \%$. 


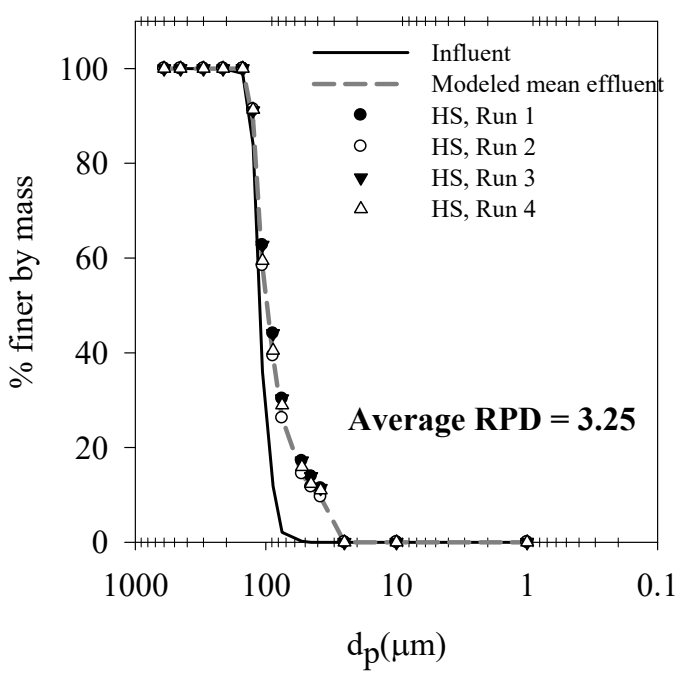

Figure 3: $\quad$ Physically measured and CFD model of scoured PSD from HS.
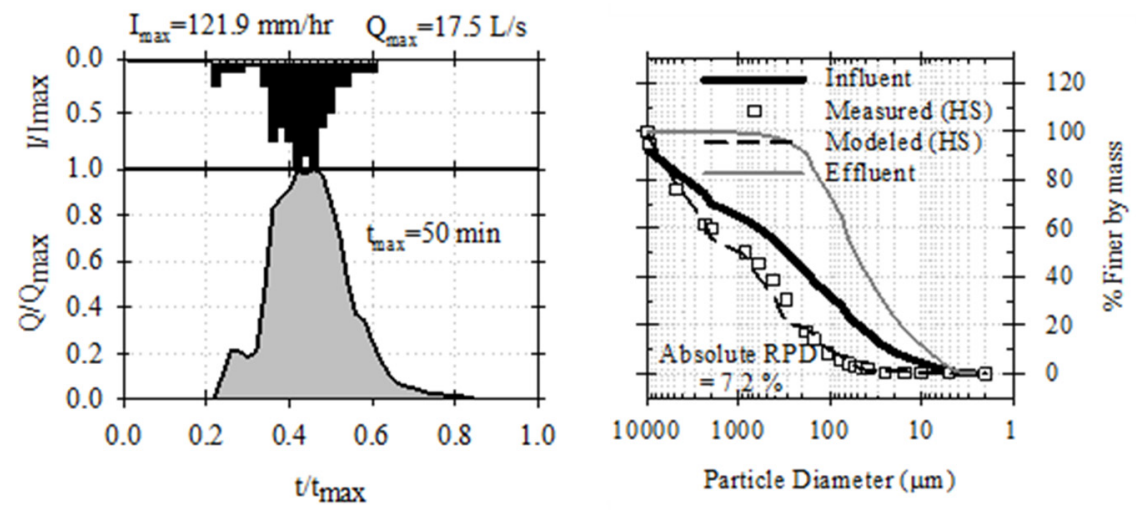

Figure 4: Hydrology with corresponding physical and CFD model of HS PSDs.

\subsection{Design and forecasting of large-scale clarifier as a storage basin}

CFD is a very effective tool for economical prototyping, optimization of design alternatives, failure forensics, retrofitting and forecasting at a wide range of temporal and spatial scales. The potential of CFD in design and forecasting is illustrated for a large-scale clarification basin with respect to sequestration of PM, TP and TN. The basin is approximately $300 \mathrm{~m}$ from north to south and 100 meters from east to west and the area, volume and aesthetic context of the basin was not altered with the retrofit. Figure 5 illustrates the velocity distribution in the basin with a retrofit design that included subsurface permeable gabion baffles and associated upper movable geosynthetic curtain associated with each of these 10 
gabions. The baffle systems can clearly be seen as linear solid areas situation in a parallel pattern from north (the arrow is pointing north) to the southern discharge end of the basin where the three box culverts are located. These CFD results utilize a mean annual $(2.3 \mathrm{yr})$ design storm and $25^{\circ} \mathrm{C}$. The profile shown is at a depth of 2 meters from the bottom of the basin which has a mean depth of 4 meters during the wet season. Velocities are relatively low in most sections; less than $0.01 \mathrm{~m} / \mathrm{s}$, a flow thalwag velocity range of: $3.0 \times 10^{-3}$ to $1.0 \times 10^{-2} \mathrm{~m} / \mathrm{s}$ and an intra-baffle velocity range of $4.0 \times 10^{-4}$ to $1.2 \times 10^{-3} \mathrm{~m} / \mathrm{s}$. The separation efficiency for TP, TN and PM for the mean annual storm event is 49, 40 and $79 \%$.

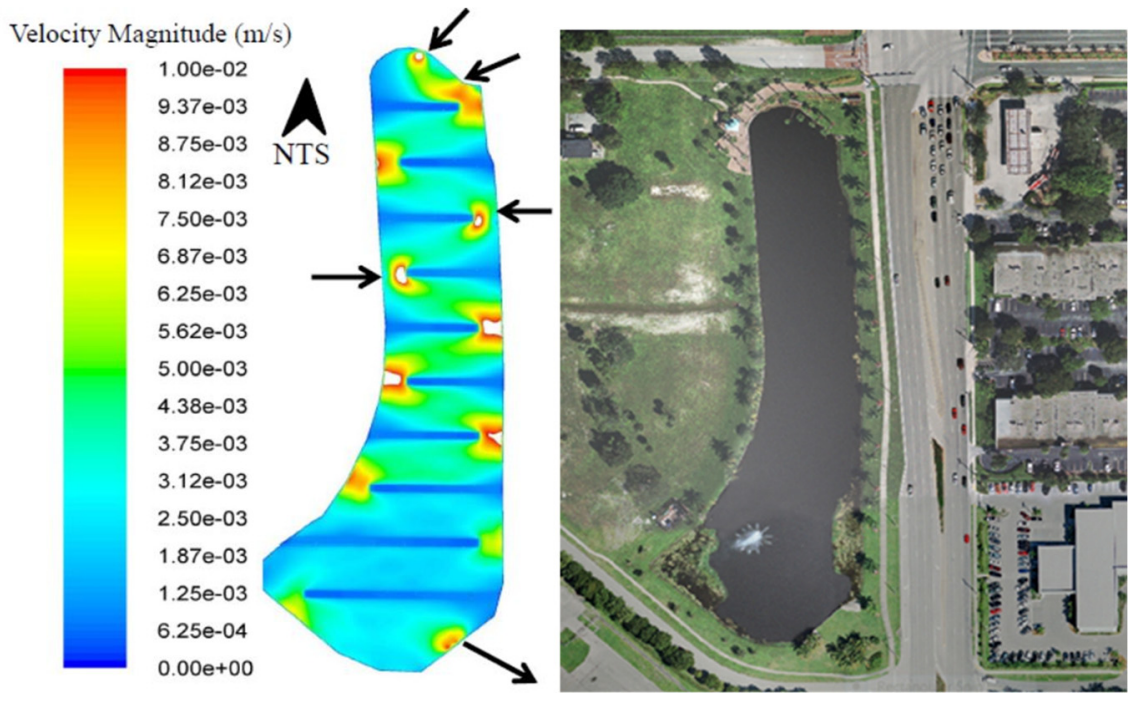

Figure 5: Velocity distribution for mean annual storm (2.33 year return) and sequestration values (separation performance as compared to influent) for TP, TN and PM for the gabion baffle design illustrated.

\section{Conclusions}

There are many configurations of unit operations to physically control the transport and fate of PM, PM-associated pathogens and PM-based chemicals which partition between the aqueous and PM phases. This study coupled physical modelling and validated CFD modelling to examine multiphase flow behaviour (separation and subsequent scour). The CFD modelling approach agreed well with physical modelling data for PM separation. Models and data results were within $10 \%$. Physical modelling results were supported by mass and volume balances. The combination of physical modelling, CFD and representative measurements of aqueous and solid phases allows better understanding and examination of BMP mechanisms. An initial foundation of controlled physical modelling of unit operations and processes for mechanistic understanding must be coupled with 
subsequent uncontrolled, unsteady and stochastic in-situ testing to provide both a mechanistic framework and knowledge of more complex in-situ behaviour, misbehaviour and maintenance of a BMP. A crucial variable that decision makers need to address is the effluent mass yield due to scour, due the reality of the expense associated with maintenance. The pre-loaded sediment depths range tested herein is generated in a range of 6 to 12 months of loadings depending on climate; suggest a maintenance cycle in terms of months not a year. Using a validated CFD model can facilitate a greater degree of accuracy than empirical approaches to examine performance, scour and maintenance requirements. Unlike black-box methods physical modelling coupled with CFD and representative measurement data allows a "BMP" to be mechanistically understood instead of a system that is simply identified as a "BMP" If a unit operation or process is put into place the unit must be maintained frequently (in terms of months) to achieve treatment performance predictions. We maintain our wastewater clarifiers or filters on a frequent basis; pumping sludge every several hours. Other than the expense of maintenance why do we think that "BMPs can go for a year or longer without maintenance? The current practice of distributed BMPs without frequent maintenance is not sustainable and careful analysis with tools such as validated CFD can demonstrate that these current practices can represent sources of PM and PM-based chemicals and pathogens. Ultimately, source control, near-source control and quantifiable maintenance practices in our urban and highway environs such as pavement cleaning combined with de-centralized hydrologic controls and regional/centralized treatment control will demonstrate sustainability.

With respect to effluent mass yield due to scour and maintenance frequency, small footprint unit operations and processes such as the HS and RCF require much more frequent maintenance than the much larger scale storage basin clarifier. For example, small manufactured units such as the HS requires maintenance on the order of months to ensure that resuspension and scour do not significantly degrade unit functionality. Furthermore the water chemistry within the HS and RCF between storms can degrade from aerobic to anaerobic within days between storms altering partitioning of metals and nutrients that can be washed out during a subsequent event. The large scale clarifier designed for volume and flow control as well as load control has a large benthic surface area where the sediment accumulated depth is small compared to the depth and volume of the basin. Maintenance is on the order of a decade or two with respect to sediment accumulation. Additionally, assimilation, burial and biological conversion/uptake are significant factors in such systems that small manufactured systems cannot replicate. Such basins are also used as aesthetic amenities in many urban areas with biological and vegetative enhancements. From a hydrologic point of view, larger surface area/volume systems allow interaction with groundwater and surface water as well as evaporation/evapotranspiration, processes that are not available to an appreciable degree for manufactured systems. Small unit operations and processes or BMPs have their beneficial niche in urban areas compared to large scale basis, however there is a commensurate requirement for frequent maintenance, on the order of months to maintain the original functionality and performance. 


\section{References}

[1] Sansalone, J.J. Physical and chemical nature of stormwater pollutants, in Wet weather flow in the urban watershed, 1st ed., edited by R. Field and D. Sullivan, CRC Press, Boca Raton, FL, USA, pp. 43-66, 2002.

[2] Kim, J.Y. and J.J. Sansalone. Event-based size distributions of particulate matter transported during urban rainfall-runoff events. Water Research, Vol. 42, No. 10-11, pp. 2756-2768, 2008.

[3] Sansalone, J.J. Adsorptive-infiltration of metals in urban drainage - media characteristics. Science of the Total Environment, Vol. 235, pp. 179-188, 2002.

[4] Huber, W.C. and R.E. Dickinson. Storm Water Management Model User's Manual, Version 4. EPA/600/3-88/001a (NTIS PB88-236641/AS). U.S. Environmental Protection Agency, Athens, GA, pp. 595, 1988.

[5] Weib, G. Vortex Separator - Proposal of a Dimensioning Method. Water Sci. and Technol., Vol. 36, No. 8-9, pp. 201-206, 1997.

[6] Paul, T.C., S.K.Sayal, V.S. Sakhuja and G.S. Dhillon. Vortex-settling basin design considerations. J. Hydraul. Eng.-ASCE., Vol. 117, No. 2, pp. 172$189,1991$.

[7] Keblin, V., M. Barrett, J. Malina and R. Charbeneau. The effectiveness of permanent highway runoff controls: sedimentation/filtration systems. CRWR Online Report 97-4. University of Texas at Austin, 1997.

[8] Dickenson J. and J.J. Sansalone. Discrete Phase Model Representation of Particulate Matter (PM) for Simulating PM Separation by Hydrodynamic Unit Operations. Environmental Science and Technology, Vol. 43, No. 21, pp. 8220-8226, 2009.

[9] Faram, M.G and R. Harwood. A method for the numerical assessment of sediment interceptors. Paper presented in 3rd International Conference on Sewer Processes and Networks, Paris, France, 2002.

[10] Tyack, J.N. and R.A. Fenner. Computational fluid dynamics modeling of velocity profiles within a hydrodynamic separator. Water Sci. Technol., Vol. 39, No. 9, pp. 169-176, 1999.

[11] Gulliver, J.S., Q. Guo and J.S.Wu. Scaling relations for manufactured stormwater BMPs. ASCE/EWRI Task Committee on Guidelines for Certification of Manufactured Stormwater Best Management Practices (BMPs). Paper presented at World Environmental and Water Resources Congress, May 12-16, Honolulu, HI, 2008.

[12] Sansalone J, S. Kuang, G. Ying and V. Ranieri. Filtration and clogging of permeable pavement. Water Research, Vol. 46, No. 20, pp. 6763-6774, 2012.

[13] Pathapati, S. and J. Sansalone. Can a Stepwise Steady Flow Computational Fluid Dynamics Model Reproduce Unsteady Particulate Matter Separation for Common Unit Operations? Environmental Science and Technology, Vol. 45, pp. 5605-5613, 2011.

[14] American Society for Testing and Materials (ASTM). Standard practice for dry preparation of soil samples for particle size analysis and determination 
of soil constants, Annual book of standards, Designation: D422-6385. Vol. 04.08, pp. 8-9, Philadelphia, 1993.

[15] Sheng, Y., G. Ying and J.J. Sansalone. Differentiation of transport for particulate and dissolved water chemistry load indices in urban source area watersheds. J Hydrol., Vol. 361, No. 1-2, pp. 144-158, 2008.

[16] Versteeg, H. and W. Malalasekera. An Introduction to Computational Fluid Dynamics: The Finite Volume Method Approach, 1st ed., Prentice Hall, London, UK, 1995.

[17] Launder, B.E. and D.B. Spalding. The numerical computation of turbulent flows. Comput. Method, Appl. M., Vol. 3, pp. 269-289, 1974. 